\title{
Metacognition research in mathematics education: from hot topic to mature field
}

\author{
Gloria Stillman · Zemira Mevarech
}

Accepted: 18 February 2010/Published online: 8 March 2010

(c) FIZ Karlsruhe 2010

Metacognition has proved a fertile field for research in education for many years, mainly inspired by the early work of Flavell on metamemory in the 1970s. Since Flavell (1976, 1979, 1981) and his colleagues initiated research into metacognition, the use of the term proliferated until there was an extensive "family of concepts that [were] often referred to generically as metacognition" (Brown, 1987, p. 109). This was mainly in the area of metacognitive research on reading but there was a growing body of early work in mathematics education as well mainly related to problem solving particularly inspired by Schoenfeld (1983, 1985, 1987) and Garofalo and Lester (1985). Problem solving remains a fruitful area for inspiration in metacognition research in mathematics education today. As Yimer and Ellerton point out in this issue, "investigating the extent to which students value problem solving and the extent to which they value themselves as problem solvers are important aspects of metacognitive research".

More recently, Flavell has been working in metacognition but in the new area of visual metacognition (see Levin, 2004) which has not as yet been taken up in mathematics education but may prove to be a new direction of research in the future. Instead, a majority of researchers in metacognitive research in mathematics education have returned to the roots of the term and share Flavell's early definition and elaborations (Desoete \& Veenman, 2006). This is not to imply that the field has been static in mathematics

G. Stillman $(\bowtie)$

Australian Catholic University, 1200 Mair Street,

Ballarat, VIC 3350, Australia

e-mail: gloria.stillman@acu.edu.au

Z. Mevarech

Bar-Ilan University, 52900 Ramat-Gan, Israel

e-mail: mevarz@mail.biu.ac.il education. On the contrary, by firmly establishing the foundations of the construct and building on these foundations, several researchers in the field have extended Flavell's work gainfully and there is a growing body of knowledge in the area.

Metacognition has often been described simply as thinking about one's own thinking. Flavell, Miller, and Miller (2002) define it as "any knowledge or cognitive activity that takes as its object, or regulates, any aspect of any cognitive activity" (p. 164). Flavell's (1979) model of metacognition and cognitive monitoring has underpinned much of the research on metacognition since he first articulated it. According to his model, a person's ability to control "a wide variety of cognitive enterprises occurs through the actions and interactions among four classes of phenomena: (a) metacognitive knowledge, (b) metacognitive experiences, (c) goals (or tasks), and (d) actions (or strategies)" (p. 906). The elements of this model have now been extended by others (e.g., elaborations of metacognitive experiences, see Efklides, 2001, 2002) or are the subject of debate (e.g., motivational and emotional knowledge as a component of metacognitive knowledge, see Op 't Eynde, De Corte \& Verschaffel, 2006). Subsequently, this has led to many theoretical elaborations, interventions and ascertaining studies in mathematics education research. Given the extent of research in the field of metacognition and mathematics education, this theme issue that includes theoretical developments and the latest empirical findings addressing developmental issues and the link to developing mathematical competencies is timely.

The issue begins with an overview by Schneider and Artelt of developments in metacognition research and theory generally. This paper reviews recent trends in research on metacognition and its relationship to the development of mathematical competencies. Major 
theoretical conceptualisations of metacognition are briefly described, followed by an overview of recent findings on developmental trends in both declarative and procedural metacognitive knowledge. Assessment of metacognition and the difficulties this entails are presented. Research findings concerning relationships among measures of declarative as well as procedural metacognitive knowledge are described. Finally, implications for training and formal instruction in educational settings are considered, based on results of innovative programs which illustrate the importance of considering metacognitive approaches in the domain of mathematics. From a developmental perspective, these authors conclude that there is evidence that metacognitive knowledge relevant for schooling usually develops through the primary years but does not reach a peak until after adolescence. However, as these authors point out there is a dearth of empirical studies that address the developmental relationships between metacognition, general intelligence and prior knowledge in a systematic manner.

Whitebread and Coltman report on evidence collected within a UK study concerning metacognition in young children in the 3- to 5-year age range within mathematical contexts. The study confirms that young children show evidence, through their talk, and their non-verbal actions, of emergent metacognitive processes, and that the nature and frequency of these processes can be influenced by pedagogical aspects of the mathematical activities.

A study by Kramarski, Weisse and Kolsher which investigated the effects of self-metacognitive questioning training on Year 3 students' (a) mathematical problem solving; (b) mathematical anxiety; and (c) on problem solving and anxiety of mathematics of higher and lower achievers is reported. The metacognitive training was based on the IMPROVE self-questioning method (Kramarski \& Mevarech, 2003). In addition, students' anxiety of mathematics was assessed. Findings indicated that the metacognitively trained students gained more than the control students in mathematical problem-solving performance. In addition, the metacognitively trained students reduced their mathematical anxiety more than the control students, particularly the lower achievers in the metacognitively trained group.

As young children are not usually aware of the limitations of their mathematical knowledge this lack of awareness is likely to present difficulties for them in effectively monitoring and regulating school tasks such as word problem solving. However, as mentioned above, there seems to be some research evidence suggesting that metacognitive skills develop in sophistication through the primary years. It is thus of interest to see if instructional programs in metacognitive skills also have a differential effect on the facilitation of problem-solving skills of students of different age groups. The effects of metacognitive instruction on Year 3 and Year 6 students' word problem solving are the subject of a paper by Mevarech, Terkieltaub, Vinberger and Nevet. Although students of both year levels benefited from the metacognitive instructional program IMPROVE, the effect size was greater for the Year 3 students. This finding confirms the potential benefit of providing young students with mechanisms such as the questioning scaffolds in the IMPROVE framework to develop their awareness of what they know and when, how and why they should apply it.

Parallel with the rethinking of the nature and the objectives of mathematics education by the researchers in the field, numerous initiatives have been introduced around the world to reform and innovate classroom practices. This has also been the case in the Flemish part of Belgium where for mathematics education there has been a deemphasizing of the teaching and practising of procedures and algorithms whilst stressing, among other things, the importance of heuristic approaches to word problem solving and the use of metacognitive skills. It is assumed that acquisition of these skills is positively correlated to students' performances on word problems and thus leads to the development of mathematical competencies. These reform-based ideas have led to a new generation of textbooks. However, as teachers and students are active interpreters of curriculum, changes in the intended curriculum do not automatically result in innovations in the implemented and thus the attained curriculum. Therefore, Depaepe, De Corte and Verschaffel were interested to investigate the extent to which heuristic and metacognitive skills are addressed in regular mathematics classrooms and how teachers' approaches towards these skills relate to students' problem-solving skills. An observational study was carried out in two Year 6 mathematics classrooms, in which the same reform-based textbook was used. This text applied a metacognitive strategy for solving mathematical application problems systematically throughout its entirety. Two teachers selected for intensive study took different approaches towards word problem solving. Also, both teachers substantially differed in the extent to which they addressed heuristic and metacognitive skills in their implementation of this same mathematical textbook. One teacher's instructional approach deliberately included many references to the metacognitive model, whilst just as deliberately the other teacher's approach did not. However, in both classrooms planning to solve the problem was almost neglected entirely. References to metacognitive skills and heuristics were almost exclusively made by teachers not students. Analyses of students' perceptions regarding the extent to which the five-stage model for application problems was referred to revealed a significant difference between both classrooms in line with the 
researchers' classroom observations. Despite this, students in both classrooms made significant progress on a word problem test over the period of the study.

Both intelligence and metacognitive skillfulness have been regarded as important predictors of mathematics performance. The role that metacognitive skills play in mathematics, however, seems to be subject to change in the early years of secondary education (i.e., when students are 13-15 years old). Metacognitive skills seem to become more general (i.e., less domain-specific) by nature (Veenman \& Spaans, 2005). Moreover, according to the monotonic development hypothesis (Alexander, Carr, \& Schwanenflugel, 1995), metacognitive skills increase with age independent of intellectual development. This hypothesis was tested in a study by Van der Stel, Veenman, Deelen and Haenen of students in the first 2 years of secondary education. Results showed that the frequency of metacognitive activities, especially those of planning and evaluation, increased with age. Intelligence was a strong predictor of mathematics performance for the 13- to 14-year-olds in the study, but less so for the 14- to 15-year-olds. Although the quality of metacognitive skills appeared to predict mathematics performance in both age groups, its predictive power was much stronger in the older group, even on top of intelligence. This evidence supports the monotonic development hypothesis. However, the various components of metacognitive skillfulness, namely, orientation, planning, elaboration and evaluation, "differ in their developmental trajectory". This is of interest to mathematics education as it shows the increasing relevance of metacognitive skills to mathematics learning with age.

Cohrs-Fresenborg, Kramer, Pundsack, Sjuts and Sommer report studies concerning the correlation of practised monitoring activities and performance in mathematics. An analysis of PISA-2000 results of German students shows that problems which demanded special competencies in metacognition were scarcely ever solved in a significant manner. In an empirical study recording students' eye movements as they attempted to problem solve, it was shown that those who had suggested inappropriate solutions had only captured a few spots during their eye movements, if any at all, which would have given hints to inconsistencies. Many students showed a striking inaccuracy when analysing. Problem-solving success was dependent on the quality of the monitoring engaged in.

Based on empirical data gathered whilst pre-service teachers engaged in non-routine mathematics problem solving, Yimer and Ellerton (2006) develop and interpret their five-phase cognitive/metacognitive model of problem solving to describe the range of cognitive and metacognitive approaches used. The five cognitive phases are engagement, transformation-formulation, implementation, evaluation and internalisation. The last of these is not a separate feature of similar cognitive/metacognitive models of problem solving by other researchers. Corresponding metacognitive behaviours have been associated empirically with each of these phases. The model caters for a variety of pathways that can be adopted during any problem-solving process by recognising that the path between these five phases is neither linear nor unidirectional. Reflection is an integral part of each category and of the model itself. Evidence of internalisation is taken by these authors to be a reflection of "the level of emerging metacognitive maturity" of the problem solver.

In conclusion, we must point out that there is much to be learnt from this international collaborative publication effort. In particular, the authors of various papers have identified a variety of possible research questions and directions for the future which will ensure that metacognition will be a fruitful field of research in mathematics education for many years to come.

\section{References}

Alexander, J., Carr, M., \& Schwanenflugel, P. J. (1995). Development of metacognition in gifted children: directions for future research. Developmental Review, 15, 1-37.

Brown, A. L. (1987). Metacognition and other mechanisms. In F. E. Weinert \& R. H. Kluwe (Eds.), Metacognition, motivation, and understanding. Hillsdale, NJ: Erlbaum.

Desoete, A., \& Veenman, M. (Eds.). (2006). Metacognition in mathematics education. New York: Nova Science.

Efklides, A. (2001). Metacognitive experiences in problem solving: metacognition, motivation, and self-regulation. In A. Efklides, J. Kuhl, \& R. M. Sorrentino (Eds.), Trends and prospects in motivation research (pp. 297-323). Dordrecht, The Netherlands: Kluwer.

Efklides, A. (2002). The systematic nature of metacognitive experiences: feelings, judgements, and their interrelations. In $\mathrm{M}$. Izaute, P. Chambres, \& P.-J. Marescaux (Eds.), Metacognition: process, function, and use (pp. 19-34). Dordrecht, The Netherlands: Kluwer.

Flavell, J. H. (1976). Metacognitive aspects of problem solving. In L. B. Resnick (Ed.), The nature of intelligence (pp. 231-235). Hillsdale, NJ: Erlbaum.

Flavell, J. H. (1979). Metacognition and cognitive monitoring: a new area of cognitive-developmental inquiry. American Psychologist, 34, 906-911.

Flavell, J. H. (1981). Cognitive monitoring. In W. Dickson (Ed.), Children's oral communication skills. New York: Academic Press.

Flavell, J. H., Miller, P. H., \& Miller, S. A. (2002). Cognitive development (4th ed.). Upper Saddle River, NJ: Prentice Hall.

Garofalo, J., \& Lester, F. (1985). Metacognition, cognitive monitoring and mathematical performance. Journal for Research in Mathematics Education, 16(3), 163-176.

Kramarski, B., \& Mevarech, Z. (2003). Enhancing mathematical reasoning in the classroom: effects of cooperative learning and metacognitive training. American Educational Research Journal, 40(1), 281-310.

Levin, D. T. (2004). Thinking and seeing: visual metacognition in adults and children. Cambridge, MA: MIT Press. 
Op 't, P., De Corte, E., \& Verschaffel, L. (2006). Beliefs and metacognition: an analysis of junior-high students' mathematicsrelated beliefs. In A. Desoete \& M. Veenman (Eds.), Metacognition in mathematics education (pp. 83-101). New York: Nova Science.

Schoenfeld, A. H. (1983). Episodes and executive decisions' in mathematical problem solving. In R. Lesh \& M. Landau (Eds.), Acquisition of mathematics concepts and processes (pp. 345395). New York: Academic Press.

Schoenfeld, A. H. (1985). Making sense of "outloud" problem solving protocols. Journal of Mathematical Behavior, 4(2), 171191.
Schoenfeld, A. H. (1987). What's the fuss about metacognition? In A. Schoenfeld (Ed.), Cognitive science and mathematics education (pp. 189-215). Hillsdale, NJ: Erlbaum.

Veenman, M. V. J., \& Spaans, M. A. (2005). Relation between intellectual and metacognitive skills: age and task differences. Learning and Individual Differences, 15, 159-176.

Yimer, A., \& Ellerton, N. F. (2006). Cognitive and metacognitive aspects of mathematical problem solving: an emerging model. In P. Grootenboer, R. Zevenbergen, \& M. Chinnappan (Eds.), Identities, cultures, and learning spaces (pp. 575-582). Adelaide, Australia: Mathematics Education research Group of Australasia. 